\title{
Use of bilateral internal iliac artery balloon occlusion as a preventative measure for a high-risk hydatidiform mole treated with surgical evacuation: A case report
}

\author{
SAHAR ABDULRAHMAN $^{1}$, MIRNA ELGHOBASHY ${ }^{2}$, JULES DYER ${ }^{3}$ and ALAA EL-GHOBASHY ${ }^{1}$ \\ ${ }^{1}$ Department of Obstetrics and Gynaecology, The Royal Wolverhampton NHS Trust, Heath Town, \\ Wolverhampton, West Midlands WV10 0QP; ${ }^{2}$ College of Medical and Dental Sciences, \\ University of Birmingham, Birmingham B15 2TT; ${ }^{3}$ Department of Intervention Radiology, \\ The Royal Wolverhampton NHS Trust, Heath Town, Wolverhampton, West Midlands WV10 0QP, UK
}

Received June 30, 2021; Accepted August 30, 2021

DOI: 10.3892/wasj.2021.124

\begin{abstract}
The present study demonstrates the use of bilateral internal iliac artery balloon occlusion (BIIABO) as a preventative measure in the management of high-risk molar pregnancies undergoing surgical evacuation of the uterus. The present study reports the case of a 27 -year-old female, gravida 1 para 0 , who presented with an ultrasound scan indicative of hydatidiform mole and a $\beta$-human chorionic gonadotropin level of 2,263,570 IU/1. Due to her pre-operative anaemia and increase levels of blood markers, she was considered as having a high-risk of haemorrhage and was thus treated with BIIABO, whilst undergoing the surgical evacuation of the uterine contents. Bilateral internal iliac artery balloons were inserted, and the uterine contents were successfully evacuated. To the best of our knowledge, this is the first study exploring the use of BIIABO as a preventative measure in the management of women with hydatidiform mole who are at risk of severe bleeding.
\end{abstract}

\section{Introduction}

Gestational trophoblastic disease (GTD) encompasses conditions ranging from hydatidiform mole (HM), to malignant disease processes, such as invasive mole, choriocarcinoma, placental site trophoblastic and epithelioid trophoblastic tumours known collectively as gestational trophoblastic neoplasia. Molar pregnancies originate from villous trophoblasts, and are characterised by trophoblastic hyperplasia and

Correspondence to: Dr Alaa El-Ghobashy, Department of Obstetrics and Gynaecology, The Royal Wolverhampton NHS Trust, Heath Town, Wolverhampton Road, Wolverhampton, West Midlands WV10 0QP, UK

E-mail: alaaelghobashy@nhs.net

Key words: balloon occlusion, gynaecology, hydatidiform mole, interventional, radiology abnormal chorionic villi resulting from the overexpression of paternal genetic material (1).

The classic features of a molar pregnancy are vaginal bleeding, hyperemesis and an enlarged uterus for the gestational age. HM most commonly affects women at extremes of child-bearing age ( $<20$ and $>35$ years of age), with those who have had a previous molar pregnancy at risk (1).

HM requires a histological diagnosis; however, an ultrasound examination is helpful in the diagnosis and has reduced the mean gestational age at presentation. The main complications of HM include malignant potential and major haemorrhage. Due to central registration in the UK at specialised trophoblastic centres and the use of $\beta$-human chorionic gonadotropin $(\beta-h C G)$ as a marker of HM, the cure rates are excellent. Suction curettage is the preferred surgical management treatment for complete hydatidiform moles (CHMs) and partial hydatidiform moles (PHMs). Medical management is only advised in the case of a PHM where foetal parts do not allow for suction. Despite the risk of bleeding associated with HM, the use of oxytocic infusions are not advised due to the potential of disseminating trophoblastic tissue (1). Minimal research has been undertaken on the use of bilateral internal iliac artery balloon occlusion (BIIABO) as a preventative measure in the management of high-risk molar pregnancies undergoing surgical evacuation. The procedure involves the placement of a balloon catheter into the internal iliac arteries bilaterally in order to reduce the haemorrhage risk (2). To the best of our knowledge, this is the first report of this treatment method for HM.

\section{Case report}

A 27-year-old Eastern European female (gravidity, 1; parity, 0) presented to the Royal Wolverhampton NHS Trust at 12/40 gestation following an abnormal booking scan. The ultrasound findings included a grossly enlarged uterus extending above the umbilicus (113 AP x127 mm), with the uterus filled with a semi solid mass containing cystic areas consistent with the 'snowstorm' appearances indicative of a HM (Fig. 1).

Upon admission, she complained of a 2-day history of minimal brown discharge, nausea and mild generalised 
abdominal pain. She was known to have irregular periods. She was not using any methods of contraception and had no previous cervical smears. The patient and her partner were not consanguineous. There was no family history of GTD. Upon examination, her uterus appeared large for gestation with the symphysis-fundal height measuring 26 weeks. The blood analysis revealed haemoglobin levels of $83, \beta$-hCG levels of 2,263,570 IU/1 and a blood group of A rhesus (Rh) negative.

Following discussion with the nearest STC, suction curettage was advised. She was considered a high-risk patient due to her anaemia upon admission and grossly elevated $\beta$-hCG levels, with a risk of having a major haemorrhage. Her case was therefore discussed with the Haematology and Interventional Radiology Department at the Royal Wolverhampton NHS Trust for a multi-disciplinary approach. Prior to surgical management, she was appropriately cross-matched, administered 2 units of packed red blood cells, and given prophylactic antibiotics in theatre.

To minimise risk of developing a haemorrhage, the interventional radiology team inserted bilateral internal iliac artery balloons. A total of $2 \times 10 \mathrm{ml} 1 \%$ lidocaine was used as a local anaesthetic. Ultrasound guided punctures of the common femoral arteries were performed by a consultant interventional radiologist with 5-French sheaths inserted into both sides (Fig. 2). Fogarty compliant balloons were inflated in the anterior division of each of the internal iliac arteries until the flow was abolished distally. She then underwent the surgical evacuation of retained products under transabdominal ultrasound guidance. The internal os of the cervix was mechanically dilated to Hegar number 12 , followed by the suction evacuation of copious amounts of products and blood, with gentle curettage under ultrasound guidance transabdominally. Following the evacuation of the uterine contents, the balloon catheters and both sheaths were removed, and arterial punctures sealed with mynx grips.

The estimated blood loss was 1.8 litres and 3 units of red blood cells were transfused in total. Anti-D prophylaxis was administered, as the patient was Rh negative. Additionally, she received an intravenous dose of tranexamic acid. The post-operatively haemoglobin levels were $94 \mathrm{~g} / \mathrm{l}$ and a Kleihauer-Betke test was undertaken to measure the degree of potential foetal-maternal haemorrhage; the results were negative. A histological examination of samples obtained during surgery confirmed a CHM. The patient was advised to use barrier contraception for 6 months following the procedure. In accordance with the UK protocol, patient was referred to the nearest specialised GTD centre for repeat 2 -weekly $\beta$-hCG tests, and was started on oral contraceptives. Her $\beta$-hCG levels markedly decreased from a pre-evacuation level of 2,263,570 to $14,101 \mathrm{IU} / 1$ on day 16 post-evacuation. The levels continued to decrease and reverted to normal 6 months later, whilst being followed-up by a STC. At 18-months following the procedure, the patient became pregnant. She gave birth at $40+1$ weeks to a healthy baby via vaginal delivery with no complications. She continues to report normal 28-day menstrual cycles.

\section{Discussion}

The use of BIIABO to minimise the risk of bleeding has been explored within obstetrics in the management of high-risk

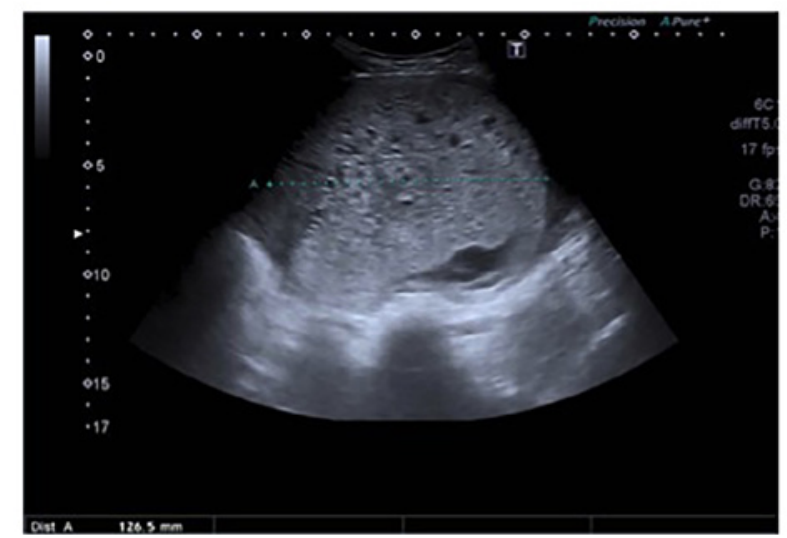

Figure 1. Transabdominal ultrasound images showing the characteristic 'snowstorm appearance' indicative of a hydatidiform mole.

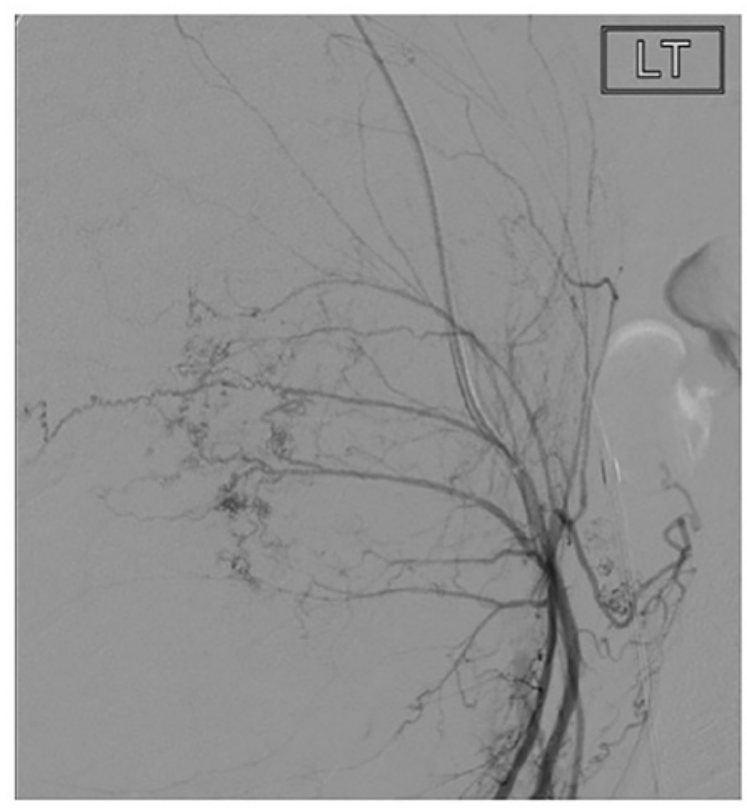

Figure 2. Angiogram of the left internal iliac artery showing the branches of the uterine artery stretched around the hydatidiform mole, prior to placement of occlusion balloons. The letters LT in the image indicate the left internal iliac artery.

caesarean sections secondary to placental invasion $(3,4)$. Despite the limited availability in the literature regarding its use in the management of HM, initial research into the use of $\mathrm{BIIABO}$ within emergency gynaecology is promising.

A single case report exists in the literature in which internal iliac arteries balloons were inserted prophylactically for a high-risk patient with HM undergoing suction curettage; however, despite a 1.1 litre blood loss, the balloons were not inflated and were removed thereafter (5). The patient however, received an oxytocin infusion following the evacuation of the uterine cavity. This raises discussion surrounding the favourability of BIIABO over alternatives, such as oxytocic infusions in achieving haemostasis.

Although much of the literature regarding BIIABO is encouraging, there have been certain safety concerns raised, most commonly thrombotic events. Internal iliac artery balloons have been associated with serious thromboembolic 
events, lower limb ischemia, puncture site complications (hematomas, false aneurysms and the dissection of femoral arteries) and other complications (6).

Anatomically, there have been suggestions that due to collateral circulation from the external iliac artery to the uterus, a common iliac artery balloon occlusion (CIABO) would provide better outcomes. Notably, a study comparing BIIABO, CIABO and no occlusion in placenta accreta managed by caesarean hysterectomy found that the mean blood loss was statistically significantly reduced only in the CIABO group $(\mathrm{P}<0.05)(4)$.

Although the National Institute for Health and Care Excellence (NICE) currently does not provide guidance on when to proceed with balloon occlusion in gynaecological or obstetric emergencies, the Royal College of Obstetricians and Gynaecologists (RCOG) has urged units to consider early or prophylactic interventional radiology as an important tool (6).

To the best of our knowledge, this is the first study to demonstrate the use of $\mathrm{BIIABO}$ as a preventative measure in the management of women with hydatidiform mole at risk of severe bleeding. The use of BIIABO in the prevention of bleeding for patients with HM undergoing suction curettage offers promise for better outcomes in the surgical management of molar pregnancies. However, due to the limited literature that exists around the topic, further extensive research is required before $\mathrm{BIIABO}$ can be considered a safe, reliable and effective intervention.

\section{Acknowledgements}

Not applicable.

\section{Funding}

No funding was received.

\section{Availability of data and materials}

Data sharing is not applicable to this article, as no datasets were generated or analysed during the current study. Data are however, available from the authors upon reasonable request.

\section{Authors' contributions}

SA was involved in the writing of the first draft of the manuscript, in the literature review, and in the editing of the manuscript. ME was involved in the writing of the first draft of the manuscript and in the editing of the manuscript. JD was involved in the clinical management of the patient and in the editing of the manuscript. AEG was involved in the clinical management of the patient, obtaining consent from the patient and in the editing of the manuscript. SA, ME, JD and AEG confirm the authenticity of all the raw data. All authors have read and approved the final manuscript.

\section{Ethics approval and consent to participate}

The patient in the present case report provided informed consent.

\section{Patient consent for publication}

The patient in the present case report provided her consent for her data to be published.

\section{Competing interests}

The authors declare that they have no competing interests.

\section{References}

1. The Royal College of Obstetricians and Gynaecologists: The Management of Gestational Trophoblastic Disease. Green-top Guideline No. 38. February, 2010.

2. Petrov DA, Karlberg B, Singh K, Hartman M and Mittal PK: Perioperative internal iliac artery balloon occlusion, in the setting of placenta accreta and its variants: The role of the interventional radiologist. Curr Probl Diagn Radiol 47: 445-451, 2018.

3. Deux JF, Bazot M, Le Blanche AF, Tassart M, Khalil A, Berkane N, Uzan $S$ and Boudghène F: Is selective embolization of uterine arteries a safe alternative to hysterectomy in patients with postpartum hemorrhage? AJR Am J Roentgenol 177: 145-149, 2001.

4. Ono Y, Murayama Y, Era S, Matsunaga S, Nagai T, Osada H, Takai Y, Baba K, Takeda S and Seki H: Study of the utility and problems of common iliac artery balloon occlusion for placenta previa with accreta. J Obstet Gynaecol Res 44: 456-462, 2018.

5. Kaneda H, Terao Y, Matsuda Y, Fujino K, Ujihira T, Kusunoki S, Kimura M, Shiraishi A, Kuwatsuru R and Takeda S: The utility and effectiveness of an internal iliac artery balloon occlusion catheter in surgery for large cervical uterine fibroids. Taiwan J Obstet Gynecol 56: 502-507, 2017.

6. The Royal College of Obstetricians and Gynaecologists: The Role of Emergency and Elective Interventional Radiology in Postpartum Haemorrhage. Good Practice No. 6. June, 2007.

This work is licensed under a Creative Commons Attribution-NonCommercial-NoDerivatives 4.0 International (CC BY-NC-ND 4.0) License. 